\title{
MINIMAL SURFACES IN TORI BY WEYL GROUPS
}

\author{
T. NAGANO AND B. SMYTH ${ }^{1}$
}

\begin{abstract}
We report a method of constructing compact minimal surfaces immersed in flat Riemannian tori of arbitrary dimension $n \geqslant 3$ by the use of the Weyl groups of compact simple Lie groups, inspired by H. A. Schwarz [5].
\end{abstract}

1. Minimal surfaces. The construction is based on two known theorems on minimal surfaces immersed in the euclidean space $\mathbf{R}^{n}$ (see Lawson [2] or Osserman [4]). Given a Jordan curve $P$ in $\mathbf{R}^{n}$, there exists a unique minimal surface $M(P)$ embedded in $\mathbf{R}^{n}$ and bounded by $P$ if $P$ satisfies the following condition (Rado's theorem):

(R) There exists an affine projection of $\mathbf{R}^{n}$ onto some 2-plane which sends $P$ bijectively onto the boundary of a convex domain $D$ in the plane.

Next we assume that $P$ is a skew-polygon satisfying $(\mathrm{R})$; thus $P$ consists of several edges. Since each edge, $e$, is a segment of a line, there corresponds the symmetry $s=s(e)$ in the line. Obviously $s$ carries $M(P)$ onto a minimal surface $s M(P)$, which is the same as $M(s P)$ by the uniqueness in Rado's theorem. Moreover the Schwarz reflection principle guarantees that the union $M(P) \cup s M(P)$ is also an immersed minimal surface; that is, the union has no singularities along the edge $e$ except possibly at the end points of $e$. We repeat this process endlessly to obtain a complete surface; more precisely, letting $S$ denote the transformation group of $\mathbf{R}^{n}$ generated by all the symmetries in the edges of $P$, we consider the orbit $S(M(P))=\cup_{s \in S} s(M(P))$, which is an immersed minimal surface without singularities except possibly at the vertices of the 1-complex $S(P)$. To eliminate this possibility, we have to choose a more special polygon.

2. Use of Weyl groups. We shall construct an example of a nice $P$, postponing a more systematic account which will require some knowledge of Weyl groups as is explained in [1]. Let $\left(e_{i}\right)_{1 \leqslant i \leqslant n}$ be an orthonormal basis for $\mathbf{R}^{n}$. Consider the set $R$ consisting of the vectors $\pm e_{i}, 1 \leqslant i \leqslant n, \pm e_{i} \pm e_{j}$, $1 \leqslant i<j \leqslant n$. Call them the roots (of $S O(2 n+1)$ ). Let $W$, the Weyl group of $R$, be the transformation group of $\mathbf{R}^{n}$ generated by the reflections in the hyperplanes through the origin 0 normal to the roots. This amounts, in this case of $S O(2 n+1)$, to the same as the group generated by the symmetries in

Received by the editors June 19, 1975.

AMS (MOS) subject classifications (1970). Primary 53A10, $22 \mathrm{E} 60$.

Key words and phrases. Minimal surface, Weyl group, torus.

'The authors acknowledge the partial support of NSF Grant GP-29662, and for the second author S.F.B. 40 at the Institute of Mathematics, Bonn, Germany. 
the lines through 0 spanned by the roots. The group $W$ is finite and leaves invariant the lattice group $L$ spanned by the roots over the integers; $L$ is the translation subgroup $\mathbf{Z}^{n}$ of $\mathbf{R}^{n}$ for our $R$. We wish to construct a polygon $P$ which satisfies these conditions: (1) P satisfies Rado's condition; (2) the vertices of $P$, viewed as position vectors, are contained in $L$; and (3) the edges of $P$ are parallel to roots. Let $P$ be the skew-polygon having the $n+1$ vertices 0 , $e_{1}-e_{2}, \ldots, e_{1}-e_{k}, \ldots, e_{1}-e_{n}$ and $e_{1}$ in this order. (For another example one may replace the last vector $e_{1}$ by $-e_{n}$.) The edges of $P$ are therefore parallel to the roots $e_{1}-e_{2}, e_{2}-e_{3}, \ldots, e_{n-1}-e_{n}, e_{n}$ and $-e_{1}$. Clearly $P$ satisfies (2) and (3). That $P$ satisfies (1) will be easily seen if one notes that condition (R) is affine in nature and that the convex hull of $P$ is the affine $n$-simplex (of which $P$ is the unique 1-subcomplex containing all the vertices, to within affine isomorphism). Observe that $L$ is a normal subgroup of $S$ and the linear part $S / L$ is the Weyl group $W$; in particular $W$ is conjugate to the isotropy subgroup of $S$ at each vertex of $P$.

We now construct an immersion $f^{\prime}: M^{\prime} \rightarrow \mathbf{R}^{n}$ of a smooth surface $M^{\prime}$, whose image is $S M(P)$ and which induces an immersion $f: M \rightarrow T=\mathbf{R}^{n} / L$ of a compact surface $M$. First we assign a subgroup $S_{(x)}$ of $S$ to each point $x$ of $M(P)$. According as $x$ is an interior point of $M(P)$, a point on an edge $e$ of $P$ different from the end points or a vertex of $P$, we denote by $S_{(x)}$ the identity $\{1\}$, the group $\{1, s(e)\}$ or the group generated by $\{s(a), s(b)\}$, $a \cap b=x$, respectively. Let $M^{\prime}$ be the quotient space of $M(P) \times S$ by the equivalence relation identifying $(x, g)$ with $(y, g h)$ under the condition $g x=$ ghy for some $h \in S_{(x)}$. The immersion $f^{\prime}$ is induced by the map: ( $g$, $x) \rightarrow g x$. That $M^{\prime}$ has no singularities at the vertices $x$ may be seen based on the fact that $S_{(x)}$ acts on the plane $p(x)$ spanned by the incident edges $a, b$ and $P$ satisfies the condition (4): the cone (or the sector) determined by $a, b$ in $p(x)$ is a fundamental domain of $S_{(x)}$ acting on $p(x)$. The left action of $S$ on itself induces an action of $S$ on $M^{\prime}$ in the obvious manner, and this gives rise to the compact surface $M=M^{\prime} / L$ as well as the $W$-equivariant immersion $f$ : $M \rightarrow T$ with $f(M)=S(M(P)) / L$. In passing let us note these facts: $f$ is a minimal immersion w.r.t. the "standard" flat metric on $T ; W$ acts effectively on $M$ and has order $n ! 2^{n}$.

3. THEOREM. For each integer $n \geqslant 3$ and each irreducible (and reduced) root system $R$ of rank $n$, there exists a minimal isometric immersion $f$ of a compact 2-dimensional Riemannian manifold $M$ into a flat Riemannian torus $T$ of dimension $n$ for which (i) the image $f(M)$ lies in no subtorus of $T$, (ii) the Gauss curvature (= the Ricci form) of $M$ is negative except possibly at a finite number of points, and (iii) the isometry group $I(M)$ has order $\geqslant n$ !, or more strongly there is an action of the Weyl group of $R$ on $M$ as a subgroup of $I(M)$ which makes $f$ equivariant.

REMARKS ON THE STATEMENT OF THE THEOREM. The metric of $T$ is not the standard one in general. By (ii) the isometry group is necessarily finite. If $M$ is orientable, then $M$ becomes a Riemann surface in the well-known way and $M$ admits a holomorphic transformation group which is isomorphic with a subgroup of $W$ of index 2.

Outline of The PRoof. Fix a Weyl chamber $C$ of $R$. Let $B(C)$ be a basis 
associated with $C$, also called a simple root system. Let $\rho_{n}$ be the highest root. Then we can choose members $\alpha_{2}, \ldots, \alpha_{n}$ of $B(C)$ such that the differences $\rho_{k}=\rho_{k+1}-\alpha_{k+1}, 1 \leqslant k<n$, defined inductively are all roots. Let $P$ be the $(n+1)$-gon having the vertices $0, \rho_{1}, \rho_{2}, \ldots, \rho_{n}$ in this order. Clearly $P$ satisfies conditions (1), (2) and (3). It follows that $L^{\prime}=S \cap L$ is a normal subgroup of $S$ such that $\mathbf{R}^{n} / L^{\prime}$ is a torus. We have $L^{\prime}=L$ by a suitable choice of $\alpha$ 's (which is necessary for the $R$ of type $E$ ). Since $W$ may not contain the inversion -1 , the linear part $S / L$ may not be isomorphic with $W$, but their extensions by $\{ \pm 1\}$ are identical. The rest goes through as before to give a minimal $W$-equivariant immersion $f: M \rightarrow T$ whose image is the compact set $S(M(P)) / L$. Induce a Riemannian metric on $M$ from $T$ by $f$. Then we have condition (iii) of the theorem. Also we have (i) since $B(C)$ is linearly independent and so is $\left(\rho_{k}\right)_{1 \leqslant k \leqslant n}$. Finally one easily infers (ii) from (i) by our previous result [3]: to wit, if a compact Riemannian manifold $M$ (of any dimension) is minimally and isometrically immersed in a flat Riemannian torus $T$, then, by passing to some finite covering of $M$, we have that $M$ is the de Rham product of a flat torus $T_{1}$ and a subspace $M_{1}$ whose Ricci form is negative definite almost everywhere and $f$ accordingly decomposes into the product of a totally geodesic immersion of $T_{1}$ and a minimal isometric immersion of $M$ both into flat tori.

REMARKs. $P$ is not unique for a given $R$ by the method above; even the change of the order of $\alpha$ 's can give a different $M$. In the case of $n=3$, the method exhausts the surfaces in [5] and [6] (with some modifications; e.g. the surface IV of [6] has branching points of order 1). There may be a more general method. Given any root which is the sum of $n$ positive roots, we can similarly construct a $P$ which satisfies (1), (2) and (3), but not (4) in general. The conclusion (iii) may not obtain either.

\section{BIBLIOGRAPHY}

1. N. Bourbaki, Eléments de mathématique, Fasc. 34, Groupes et algèbres de Lie, Chaps. 4, 5, 6, Actualités Sci. Indust., no. 1337, Hermann, Paris, 1968. MR 39 \# 1590.

2. B. Lawson, Lectures on minimal submanifolds, Inst. Mat. Pura Appl., Rio de Janeiro, 1973.

3. T. Nagano and B. Smyth, Minimal submanifolds in flat tori, Comment. Math. Helv. 50 (1975), 249-265.

4. Robert Osserman, A survey of minimal surfaces, Van Nostrand Reinhold, New York, 1969. MR 41 \#934.

5. H. A. Schwarz, Gesammelte mathematische Abhandlungen, Vol. I, Springer-Verlag, Berlin, 1890; English transl., Chelsea, New York.

6. B. Stessmann, Periodische Minimalflächen, Math. Z. 38 (1934), 417-442.

Department of Mathematics, University of Notre Dame, Notre Dame, Indiana 46556 Institute of Mathematics, University of Bonn, Bonn, Germany 